\title{
Vimentin Positive Primary Thyroid Diffuse Large B-Cell Lymphoma: Cytological Diagnosis of Two Cases
}

\author{
Swati Bhardwaj*, Charanjeet Ahluwalia and Ashish Kumar Mandal \\ Department of Pathology, Vardhman Mahavir Medical College \& Safdarjung Hospital, New Delhi, India
}

\begin{abstract}
Primary thyroid lymphomas are rare tumours of thyroid accounting for 5\% of all thyroid tumours. Diffuse large B cell lymphoma is one of the most common type of primary thyroid lymphoma. Here we are presenting two rare cases of primary thyroid lymphoma. The first case is of a sixty year old female who presented with a rapidly enlarging thyroid mass, causing compressive symptoms. Radiological investigations revealed deviation and compression of trachea by the mass. Fine needle aspiration was performed, and routine as well as liquid based cytology smears were prepared. Smears revealed features of diffuse large B cell lymphoma of thyroid. Immunocytochemistry was performed that confirmed the diagnosis, and also revealed vimentin positive nature of the neoplasm, that has been shown to confer some degree of resistance to chemotherapy. A tru cut biopsy was performed that reconfirmed the diagnosis, and also helped in determining Ki67 proliferation index of the tumour that determines the aggressive nature of non-Hodgkin's lymphomas. The second case is of a seventy year old woman who presented with a rapid enlargement of thyroid over a period of three months, leading to difficulty in breathing and dysphagia. Fine needle aspiration was performed that showed features of immunoblastic type of DLBCL. The diagnosis was confirmed by immunocytochemistry, which also showed vimentin positivity. These two cases are important for their rarity, especially being vimentin positive, diagnosed on cytology with the aid of immunocytochemistry.
\end{abstract}

Keywords: Thyroid, Cytology, FNAC, Lymphoma, Diffuse Large B Cell Lymphoma, Immunocytochemistry.

\section{Introduction}

Primary thyroid lymphoma is a rare entity and accounts for only $5 \%$ of all thyroid malignancies and approximately $3 \%$ of all non-Hodgkin's lymphoma. ${ }^{[1,2]}$ There is an overall female predominance with a female: male ratio of $3: 11^{[3,}$ ${ }^{4]}$ They typically occur in middle to old aged individuals. ${ }^{[5]}$ Most thyroid lymphomas are of B cell origin. ${ }^{[6]}$ The most common presentation of thyroid lymphoma is a rapidly enlarging, painless goitre. Other symptoms such as dyspnoea, dysphagia, and hoarseness may arise as a result of the pressure effects of the mass. Rarely, stridor or superior vena cava obstruction can occur. Cervical lymphadenopathy is present in the majority of cases. ${ }^{[7]}$ The underlying pathogenesis of primary thyroid lymphoma is not exactly known, but is attributed to chronic antigenic stimulation leading to the development of intra-thyroid lymphoid tissue as is seen in Hashimoto's thyroiditis. This also explains why the risk of development of primary thyroid lymphoma is $40-80$ times higher in patients of Hashimoto's thyroiditis. ${ }^{[4]}$

Fine needle aspiration cytology plays a vital role in diagnosing thyroid lesions. Since biopsy has a minor role to play in thyroid, the onus lies on FNA alone to establish a diagnosis for adequate patient management. The management of DLBCL thyroid is non-surgical and relies on chemo-radiotherapy. Therefore, it is important to evade surgery by establishing diagnosis on FNA beforehand.

\section{Case Reports}

CASE 1: A sixty year old female with no previous history of thyroid complaints, presented with a rapidly enlarging thyroid swelling, over a period of three months (Figure-1). It was accompanied by pressure symptoms of dysphagia. There was no history of any hyperthyroid or hypothyroid symptoms. On examination, the patient had anterior neck swelling measuring $11 \times 7 \times 6 \mathrm{~cm}$. The swelling moved little with deglutition owing to its large size. There was no palpable cervical lymphadenopathy. The patient had normal vitals, normal general physical examination and no tremors were noted. Ultrasound neck showed bilateral thyroid lobe enlargement. There was decreased echo pattern in both lobes with multiple echogenic strands, with few subcentimetric lymph nodes in level III. Features were suggestive of thyroiditis (Hashimoto's disease). The patient then underwent $\mathrm{CT}$ scan of the neck that revealed diffuse enlargement of the thyroid gland, compressing and displacing the larynx, vocal cord, and cricothyroid membrane (Figure-2). Few enlarged lymph nodes were noted on the left side of the neck, measuring $7 \mathrm{~mm}$ to $9 \mathrm{~mm}$.

Fine needle aspiration was performed from two sites, owing to the large size of the swelling. Conventional smears were prepared and material for immunocytochemistry was fixed in cold acetone. Liquid based cytology was 
also done. Conventional Giemsa stained smears were moderately cellular, showing monomorphic cells present mainly singly and a few small dyscohesive clusters against a haemorrhagic background (Figure-3a). The individual cells were large, exhibiting high degree of pleomorphism, high nucleo: cytoplasmic ratio, and scant cytoplasm. Most cells showed prominent nucleoli. Brisk mitoses, including atypical forms were noted. A differential diagnosis of diffuse large B cell lymphoma and anaplastic carcinoma were kept.

Immunocytochemistry was performed to establish a definitive diagnosis. The cells were positive for LCA, Bcl6 , and Vimentin and negative for CK (Figure-3b-3d). The smears also showed the presence of TTF-1 positive cells confirming the origin from thyroid. Thus, a diagnosis of diffuse large B cell lymphoma was established.

A tru cut biopsy was performed to reconfirm the diagnosis. Histopathology revealed features of diffuse large B cell lymphoma, which was confirmed on immunohistochemistry. (Figure-4).

The patient was started on chemo radiotherapy and is doing well now.

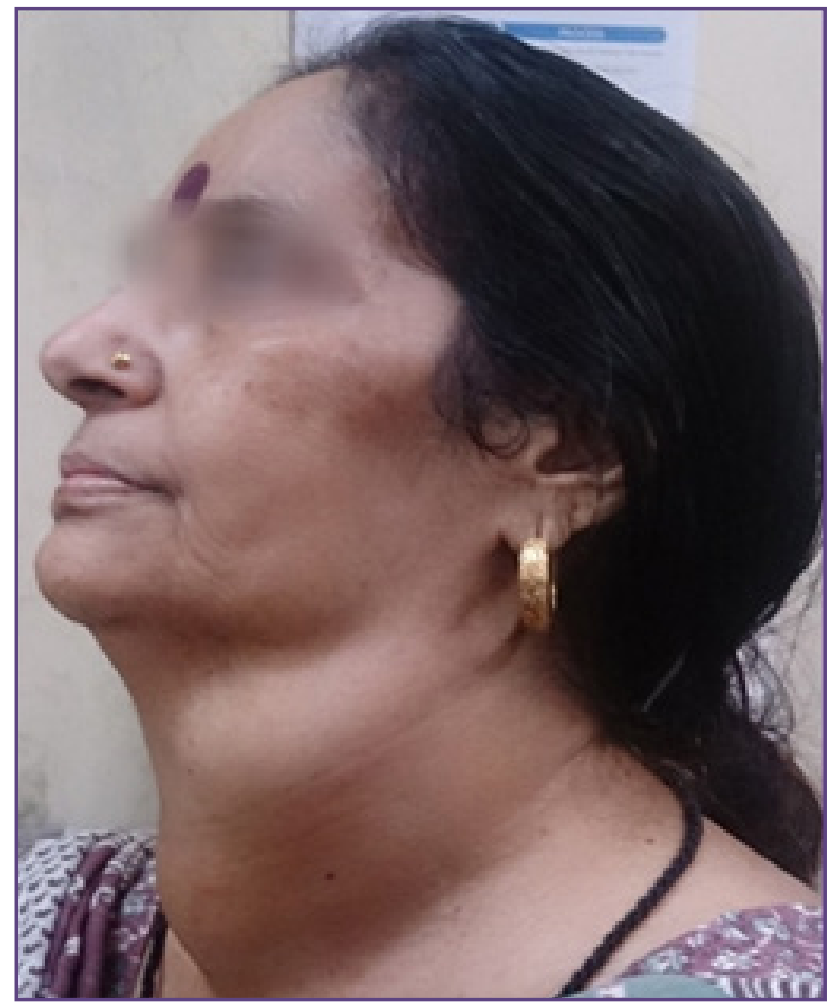

Fig. 1: Diffuse anterior neck swelling, case 1.
CASE 2: A seventy year old lady, housewife by profession presented with a rapidly enlarging thyroid swelling that increased in size over a period of five months (Figure 5a). There was history of difficulty in swallowing, and breathing present since past 1 month. There was no history of hyperthyroid or hypothyroid symptoms. The patient also had loss of weight over the past one month. On examination, the swelling was soft to firm, measuring $9.5 \times 10 \mathrm{~cm}$. It moved little with deglutition. There was no cervical lymphadenopathy. Ultrasound showed diffuse thyroid swelling, involving both lobes. CECT neck revealed diffuse thyroid enlargement, compressing and displacing trachea to one side. Retrosternal extension was also present. There was no cervical lymph node enlargement.

FNAC was performed from two sites, owing to the large size of the swelling. Smears were hypercellular, showing lymphoid cells scattered singly. The cells exhibited moderate degree of pleomorphism and a high mitotic rate. (Figure 5b)

Immunocytochemistry was performed, that showed the cells to be LCA, and BCL6 positive. They were negative for $\mathrm{CK}$ and CD20. These cells were also positive for Vimentin. Thus a diagnosis of diffuse large B cell lymphoma, immunoblastic type was made.

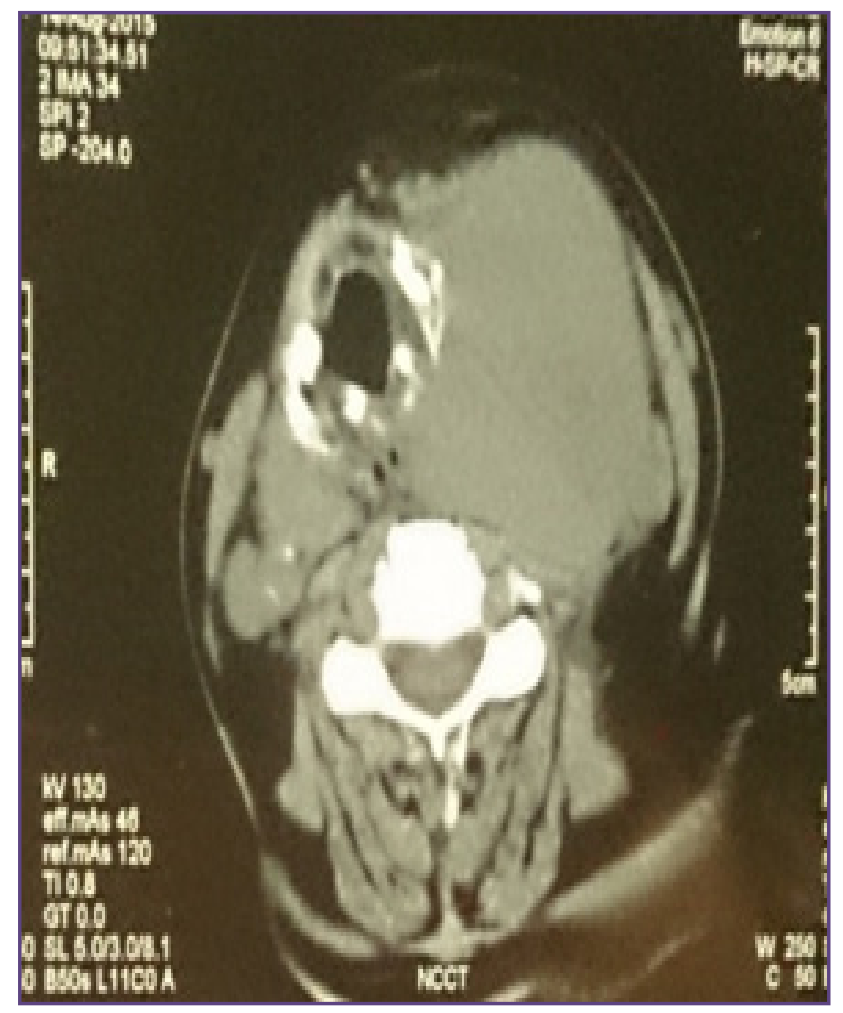

Fig. 2: CT picture shows tracheal displacement by thyroid swelling 


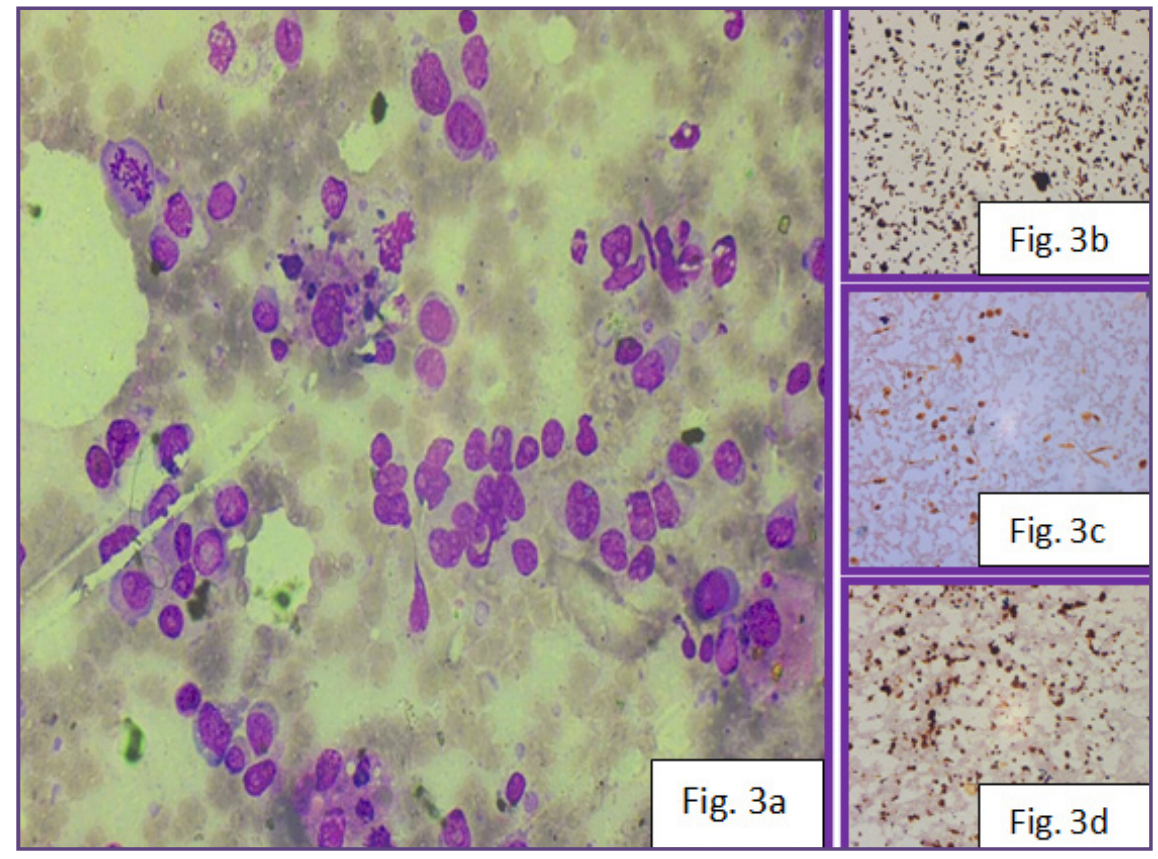

Fig. 3a :Giemsa stained smears shows scattered large, pleomorphic lymphoid cells with prominent nucleoli and brisk mitoses, 20X. Figures 3b, 3c, 3d: Immunocytochemistry showing positivity for LCA, Bcl 6 and Vimentin respectively, 10X.

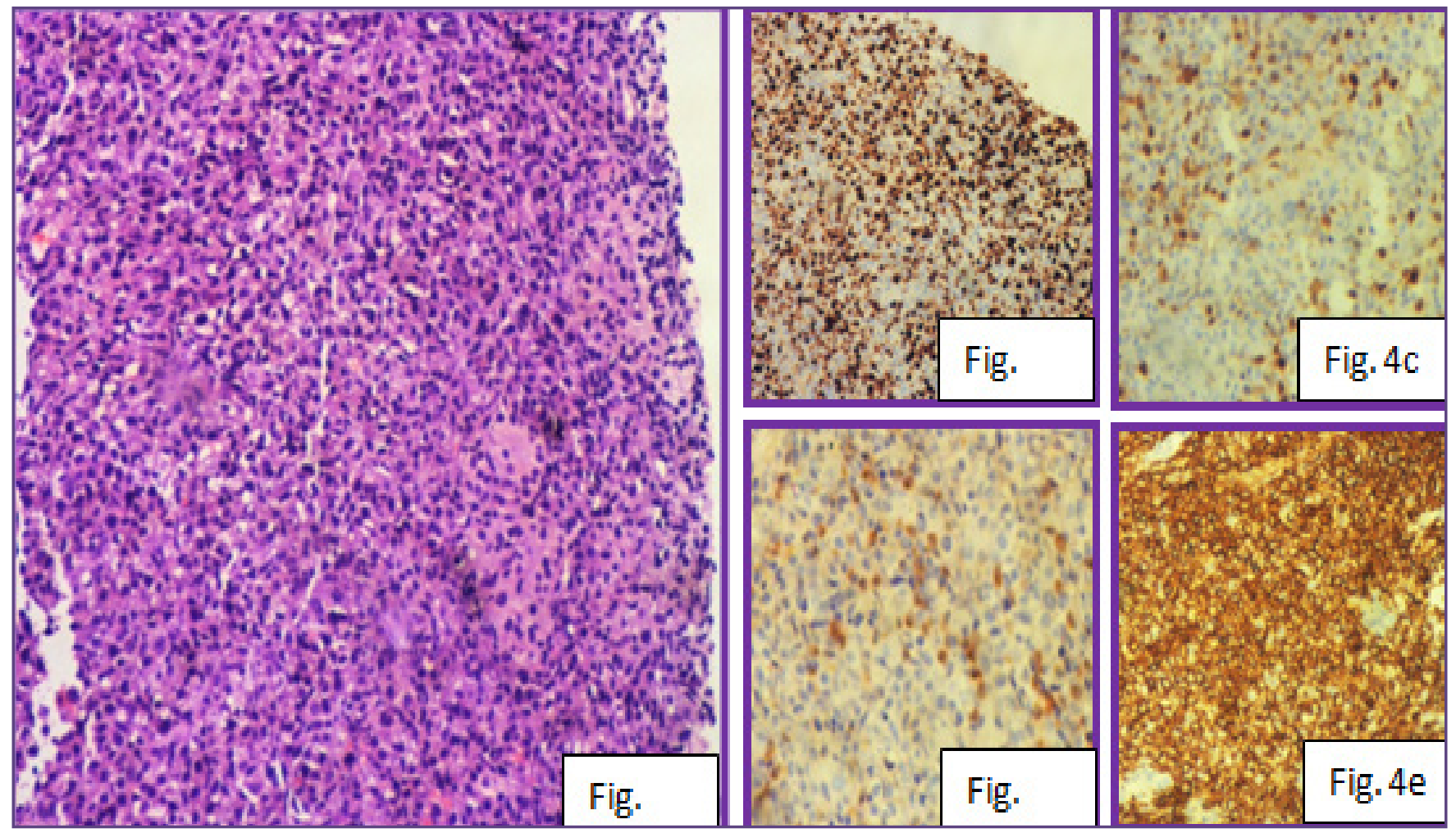

Fig. 4a: H\&E stained tru cut biopsy showing monomorphic lymphoid cells with high degree of pleomorphism, 20X. Figure 4b, 4c, 4d, 4e: Immunohistochemistry showing positivity for Ki67, Bcl-2, MUM-1 and CD-20 respectively, 20X. 


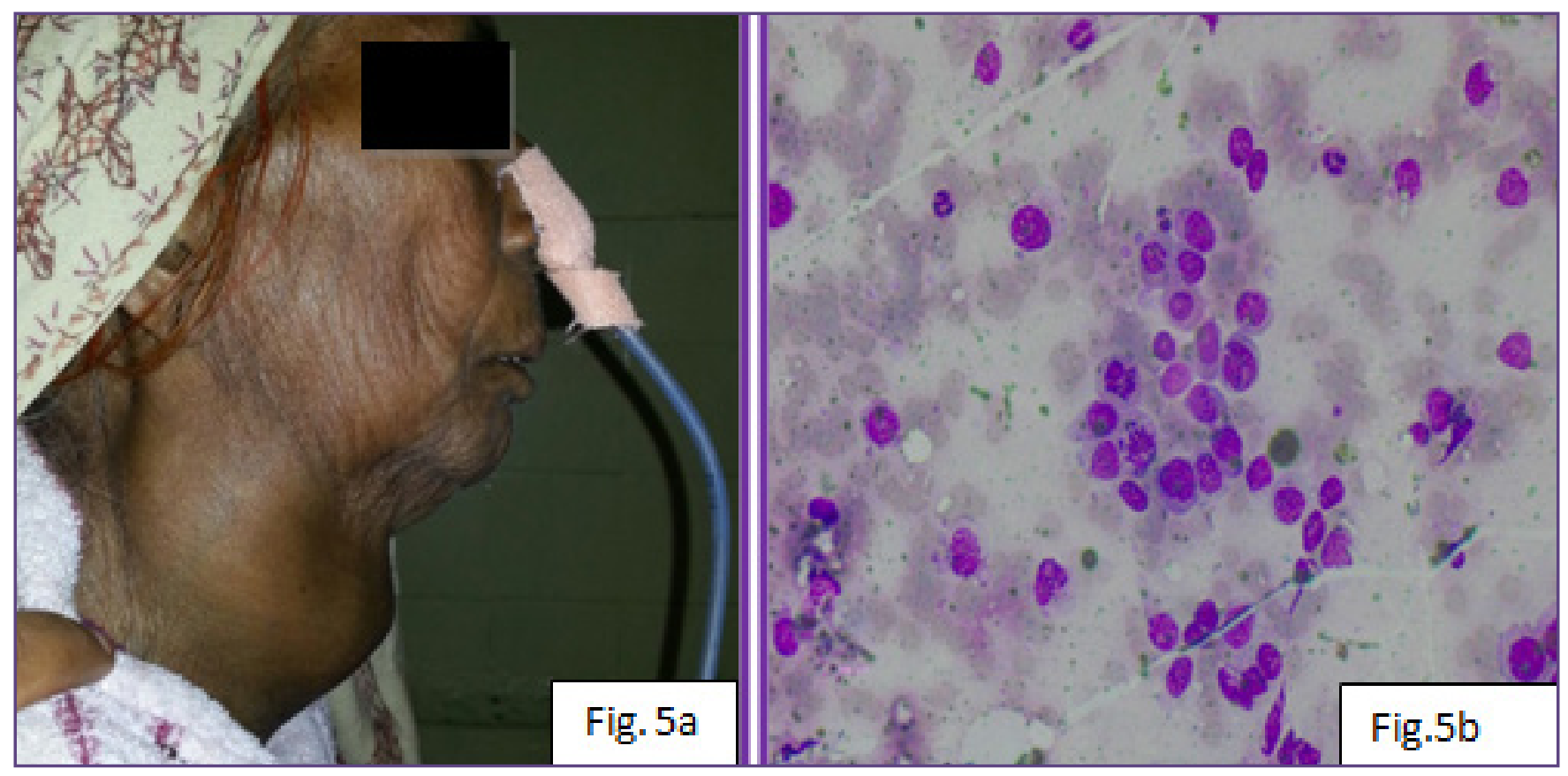

Fig. 5a: Diffuse anterior neck swelling, case 2. Figure 5b: Case 2, Giemsa stained smear showing high cellularity of lymphoid cells with prominent nucleoli and mitotic figures, $20 \mathrm{X}$.

\section{Discussion}

These two cases present an example of a rare thyroid neoplasm, diagnosed primarily by FNAC and reconfirmed on histopathology. On FNA smears, DLBCL of thyroid can be difficult to distinguish from anaplastic carcinoma thyroid. Both present with a rapidly enlarging thyroid swelling clinically, with a somewhat overlapping cytological picture of large, atypical cells with large, pleomorphic nuclei, and prominent nucleoli. It is essential to clearly and accurately distinguish the two as both require drastically different treatment. While anaplastic carcinoma is treated by radical surgery, DLBCL does not need surgery as radiotherapy and chemotherapy suffice. Differentiation between the two can be done by cytology and immunocytochemistry.

In the first case, the diagnosis had already been established on cytology, thus guiding the clinical decision against surgical management. However, a tru cut was done for a reconfirmation and for determining the ki67 proliferation index of the tumour. According to literature, a higher Ki67 proliferation index indicates a poor prognosis due to aggressive disease course. ${ }^{[8]}$ In our case, the ki67 index was about 50\%. The mean Ki67 proliferation index differs by type of lymphoma. A cut off value of $45 \%$ can help differentiate indolent from aggressive Non Hodgkin lymphoma. ${ }^{[8]}$ In diffuse large B cell lymphoma, a cut off value of $75 \%$ can distinguish patients with a good and bad prognosis, when combined with low IPI score and bulky disease. ${ }^{[8]}$
Diffuse large B cell lymphomas conventionally undergo CHOP chemotherapeutic regimen. In about $40 \%$ cases of DLBCL, development of resistance to $\mathrm{CHOP}$ regimen causes treatment failure. ${ }^{[9]}$ Among the various causes investigated, vimentin positivity was found to be one implicated in causing greater tumour invasiveness and resistance to therapy. Maxwell et al have described that activation of an Akt-14-3-3 $\zeta$ signalling pathway plays a role in promoting a multidrug-resistant phenotype associated with a vimentin-dependent invasive behaviour in DLBCL cells. [9] Thus our cases, are examples of uncommonly observed vimentin positivity, indicating poor response to chemotherapy.

Both of the patients were treated with a combination of chemo and radiotherapy and are doing well.

\section{Conclusion}

Cytology combined with other ancillary techniques such as immunocytochemistry plays a very important role in diagnosing thyroid lesions. This case report highlights the diagnostic importance of FNAC in diagnosing even rare lesions such as thyroid lymphoma. The use of immunocytochemistry enabled accurate typing of lymphoma, thereby facilitating early treatment with minimal intervention. These two cases are also important for their rarity, and uniqueness. 


\section{Reference}

1. Ansell SM, Grant CS, Habermann TM. Primary thyroid lymphoma. SeminOncol. 1999;26:316-323.

2. Green LD, Mack L, Pasieka JL. Anaplastic thyroid cancer and primary thyroid lymphoma: A review of these rare thyroid malignancies. J Surg Oncol.2006;94:725-736.

3. Graff-Baker A, Roman SA, Thomas DC, et al. Prognosis of primary thyroid lymphoma: Demographic, clinical, and pathological predictors of survival in 1,408 cases. Surgery. 2009;146:1105-1115.

4. Pederson RK, Pederson NT. Primary non-Hodgkin's lymphoma of the thyroid gland: A population based study. Histopathology.1996;28:25-32.

5. Foppiani L, Secondo V, Arlandini A, Quilici P, Cabria M, Del Monte P. Thyroid lymphoma: a rare tumor requiring combined management.Hormones (Athens). 2009; 214-8.
6. Gupta N, Nijhawan R, Srinivasan R, Rajwanshi A, Dutta P, Bhansaliy A, Sharma SC. Fine needle aspiration cytology of primary thyroid lymphoma: a report of ten cases. Cytojournal. 2005;20:21.

7. Sasai K, Yamabe H, Haga H, et al. Non-Hodgkin's lymphoma of the thyroid. A clinical study of twenty two cases. Acta Oncol.1996;35:457-462.

8. Li J, Hu R, Liao AJ, Shi HY, Yan W, Liu ZG. Ki67 proliferative index in non-Hodgkin's lymphoma and its clinical significance. Zhongguo Shi Yan Xue Ye XueZaZhi. 2011;19(4):935-9.

9. Maxwell SA, Cherry EM, Bayless KJ. Akt, 14-3-3 , and vimentin mediate a drug-resistant invasive phenotype in diffuse large B-cell lymphoma. Leuk Lymphoma. 2011 May;52(5):849-64.

*Corresponding author:

Dr. Swati Bhardwaj, 289, Sector-38, Gurgaon-122001, Haryana, India.

Phone: +91 0124-2200289, +919811859747.

Email: swat.bhardwaj@yahoo.com

Date of Submission : 03.11.2016

Date of Acceptance : 03.04.2017

Financial or other Competing Interests: None.
Date of Publication : 29.05.2017 